\title{
Modelo conceptual y tecnológico para apoyo a niños con discalculia
}

\author{
María Alejandra Hurtado Parra \\ Néstor Darío Duque-Méndez
}

\section{Resumen}

Usualmente en los ambientes educativos, tanto virtuales como presenciales, las actividades y estrategias de aprendizaje son generalizadas y buscan atender multimodal y homogéneamente a todos los estudiantes, sin reconocer las diferencias existentes. En el caso particular de la enseñanza primaria, los ambientes educativos involucran estudiantes con diversas características y ritmos cognitivos, entre ellos niños con dificultades de aprendizaje, que se ven afectados con actividades de enseñanza y aprendizaje únicas y para todos.

Los niños en edad escolar que presentan dificultades de aprendizaje pueden retardar su proceso académico, pues requieren de actividades de aprendizaje acordes a su situación. Si no se identifican y atienden a tiempo estas dificultades el día a día de los niños puede verse afectado negativamente, no solo en lo académico sino en lo social y personal.

El artículo presenta un modelo conceptual y un entorno informático educativo basado en actividades educativas encaminadas a niños con dificultades de aprendizaje relacionadas con la matemática, que permita la detección temprana y así mismo apoyen las estrategias específicas para el nivel de dificultad encontrada, permitiendo realizar el seguimiento de la evolución de cada estudiante.

Palabras clave: Discalculia. Dificultades de aprendizaje de la matemática. Habilidades matemáticas básicas. Identificación temprana.

\section{Conceptual and technological model to support children with dyscalculia}

\section{Abstract}

Usually in educational environments, both virtual and face-to-face, the activities and learning strategies are generalized and seek to address 
multimodal and homogeneous all students, without recognizing the existing differences. In the particular case of primary education, educational environments involve students with diverse characteristics and cognitive rhythms, among them children with learning difficulties, who are affected with unique teaching and learning activities for all.

Children of school age who have learning difficulties may delay their academic process, since they require learning activities according to their situation. If these difficulties are not identified and attended to on time, children's day-to-day activities can be adversely affected, not only in the academic but also in the social and personal aspects.

The article presents a conceptual model and an educational computer environment based on educational activities aimed at children with learning difficulties related to mathematics, which allows early detection and also supports specific strategies for the level of difficulty encountered, allowing the monitoring of the evolution of each student.

Keywords: Dyscalculia. Difficulty in learning mathematics. Abilities simple math. Early identification.

\section{Modelo conceitual e tecnológico de apoio às crianças com discalculia}

\section{Resumo}

Geralmente em ambientes educacionais, tanto virtuais quanto presencial, as atividades e estratégias de aprendizagem são generalizadas e buscam abordar multimodais e homogêneos todos os alunos, sem reconhecer as diferenças existentes. No caso particular do ensino fundamental, os ambientes educacionais envolvem alunos com características diversas e ritmos cognitivos, dentre eles crianças com dificuldades de aprendizagem, que são afetadas com atividades únicas de ensino e aprendizagem para todos.

As crianças em idade escolar que apresentam dificuldades de aprendizagem podem atrasar seu processo acadêmico, uma vez que requerem atividades de aprendizagem de acordo com sua situação. Se essas dificuldades não forem identificadas e atendidas no prazo, as atividades cotidianas das crianças podem ser prejudicadas, não apenas no aspecto acadêmico, mas também nos aspectos social e pessoal.

$\mathrm{O}$ artigo apresenta um modelo conceitual e um ambiente computacional educacional baseado em atividades educacionais voltadas para crianças com dificuldades de aprendizagem relacionadas à matemática, o que permite a detecção precoce e também suporta estratégias específicas para 
o nível de dificuldade encontrado, permitindo o acompanhamento da evolução de cada aluno.

Palavras-chave: Discalculia, dificuldades de aprendizagem das matemáticas, habilidades matemáticas básicas, identificação precoce.

\section{Introducción}

A lo largo del ciclo académico los estudiantes desarrollan su competencia matemática, demostrando ciertas habilidades que con el tiempo se van perfeccionando, pero en el caso de algunos estudiantes esas habilidades no se desarrollan de una forma exitosa, teniendo consecuencias en el proceso académico.

Según Rubo (2007), una de las mayores dificultades a las que se enfrenta hoy en día el sistema educativo es la deserción y el fracaso escolar ocasionado por bajo rendimiento académico, dificultades para acceder a la construcción del conocimiento y desmotivación frente al aprendizaje, especialmente en los primeros años académicos del estudiante, y que es allí donde se inicia la construcción de los procesos lógicos matemáticos que son fundamentales para aprendizajes futuros (RUBO, 2007).

En algunos casos, el origen del fracaso escolar no está asociado directamente con el interés, curiosidad, comportamiento, motivación o actitud en clase, si no por dificultades en las áreas del aprendizaje primarias, asociadas a dificultades en procesos cognitivos como la atención, percepción o memoria. Un estudiante con dificultad de aprendizaje, en especial con una dificultad específica de aprendizaje de la matemática (DAM), tiene un coeficiente intelectual promedio, pero al no identificar ni tratar dicha dificultad, los niños suelen tener bajo rendimiento académico, retrasos educativos, entre otras situaciones.

Es importante tener presente que se pueden llegar a generar conflictos dentro del aula de clase, cuando un docente no tiene las estrategias y prácticas adecuadas para atender a los niños con dificultades de aprendizaje de la matemática, posiblemente porque no se encuentra preparado o porque no cuenta con los instrumentos necesarios. 
Las TIC pueden apoyar la labor del docente y a los estudiantes que presentan DAM, llegando a ser importantes herramientas para la identificación de estas dificultades al momento de aprender las matemáticas y para realizar la intervención necesaria ofreciendo contenidos académicos y actividades pedagógicas correspondientes a la necesidad de cada estudiante, para su proceso de aprendizaje y contribuyendo a que se disminuya el fracaso en el cumplimiento de los logros establecidos por el docente.

Al realizar revisión de literatura, se observan algunas iniciativas para la identificación de las DAM tomando en cuenta diferentes enfoques. Sin embargo, la mayoría de herramientas tecnológicas consideran sólo algunas características de importancia para que la identificación se realice de forma apropiada, dejando como posibilidad una inadecuada identificación a los estudiantes evaluados. En otros casos, solo se orientan a proponer actividades de aprendizaje diversas sin una evaluación de los avances específicos.

Se propone un modelo para realizar identificación de las DAM, por medio de una prueba basada en actividades académicas asociadas a diferentes elementos. Dicho modelo presenta un enfoque por grados académico, categorías, habilidades cognitivas y niveles cognitivos, que ayudan a la construcción de las actividades académicas para prueba DAM y posterior soporte para apoyar las falencias detectadas.

El modelo se concreta en un entorno informático educativo llamado Diamante, que entrega a los estudiantes la prueba para identificación de las DAM, de acuerdo a los grados académicos propuestos $\left(1^{\circ}, 2^{\circ}\right.$ y $3^{\circ}$ de primaria). Este proceso permite al docente realizar una identificación de las fortalezas y debilidades, para así generar un plan de trabajo que ayude a la mejora de las debilidades encontradas.

Con el fin de analizar los resultados arrojados por la prueba de acuerdo a cada nivel atendido e identificar casos de estudiantes con DAM, se evaluaron, por medio de este entorno informático, estudiantes de grado primero de primaria del Colegio Aspaen 
Los Cerezos de la ciudad de Manizales-Colombia y estudiantes de casos individuales de diferentes colegios. La aplicación de este entorno informático permitió evidenciar la importancia de aplicar el modelo, no solo para realizar una entrega de resultados, sino para acompañar a los docentes en la detección temprana de las DAM en el aula de clase y orientar así a los estudiantes en aras de fortalecer su proceso académico.

Este artículo está organizado de la siguiente manera, la sección siguiente se orienta a concepto relacionado con las dificultades específicas de aprendizaje en la matemática; en el tercer apartado se exponen algunos trabajos relacionados; a continuación, se presenta el modelo para la identificación de las DAM y se expone el entorno informático educativo "Diamante" con su respectiva validación; por último, se presentan las conclusiones y el trabajo futuro.

\section{Dificultades específicas de aprendizaje de la matemática (DAM)}

Las matemáticas elementales, junto con la lectoescritura, hacen referencia a los aprendizajes instrumentales básicos que realizan los estudiantes en los primeros años académicos. Al adquirir el conocimiento matemático, pueden desenvolverse no sólo en el aula de clase sino en muchas situaciones de la vida cotidiana (ANDALUCÍA, 2012, p. 1), pero en algunas circunstancias se encuentran inconvenientes para el desarrollo de dicho conocimiento, generando una dificultad específica de aprendizaje de la matemática también llamada discalculia, definida como "dificultades que presentan estudiantes de inteligencia normal pero que rinden por debajo de su capacidad en tareas de cálculo y de solución de problemas" (ROMERO PÉREZ \& LAVIGNE CERVÁN, 2004, p. 75).

Los bajos logros aritméticos se han atribuido especialmente al déficit en las capacidades cognitivas generales, como la memoria de trabajo y la función ejecutiva (BUTTERWORTH, 2011, p. 252). En este orden de ideas, es relevante examinar el funcionamiento 
de distintas habilidades cognitivas ya que estas subyacen a los déficits aritméticos, teniendo en cuenta así habilidades intelectuales, ejecutivas, de razonamiento y de atención (IGLESIAS SARMIENTO, 2008, p. 22).

La discalculia, afecta a alrededor del 5\% de los niños en edad escolar, y ha recibido menor atención de investigadores frente a otras dificultades de aprendizaje, por ejemplo, la dislexia. En dos tercios de los niños afectados, la discalculia se asocia con otro trastorno del desarrollo como la dislexia, el trastorno por déficit de atención, el trastorno de ansiedad, el trastorno visual y espacial o la privación cultural (RAPIN, 2016, p. 13).

Son múltiples las causas que pueden desencadenar este tipo de dificultades, entre ellas, la falta de motivación por las matemáticas, la baja autoestima, la alta ansiedad, las creencias, actitudes y expectativas, sin menospreciar la influencia que ejercen diferentes factores emocionales sobre el aprendizaje de esta materia (DÍAZ, 2014). En algunos casos, pueden ser generadas por disfunción del sistema nervioso central. Generalmente, para ser identificadas deben afectar al rendimiento escolar, laboral o personal, así como generar problemas en lo que a la adaptación social, personal, escolar, familiar, laboral o comunitaria se refiere (BLANCO PÉREZ, 2009).

\section{Trabajos relacionados}

Las dificultades específicas de aprendizaje en las matemáticas son frecuentes y es importante buscar el mayor número de estrategias que faciliten la respuesta a las necesidades que desencadenan dicha dificultad. Algunas de esas estrategias exigen buscar nuevos recursos, entre ellos los tecnológicos y concretamente recursos informáticos, que pueden facilitar el aprendizaje del alumno, aprovechando las determinadas cualidades de las que disponen (SEVILLANO \& ANGELES, 2000). Existen herramientas para el tratamiento de la discalculia en el aula de clase, que ayudan a los estudiantes de primaria a realizar de actividades que le permitan 
avanzar en su proceso de adaptación y ganar fortalezas para un desarrollo académico adecuado.

En la búsqueda de información, se han encontrado herramientas tecnológicas que realizan apoyo a los estudiantes con dificultades de aprendizaje en la matemática, de las cuales se exponen algunas a continuación:

- Juan García Moreno (2010) desarrollo una herramienta multiplataforma que apoya a los docentes involucrados en enseñanza de la matemática mediante recursos educativos. Concreta de manera práctica y gráfica las líneas metodológicas utilizadas en el aula de clase que permiten a los estudiantes una interacción efectiva con los temas propuestos por el docente, pero no involucra a estudiantes con dificultades específicas de aprendizaje de la matemática.

- Daniel González de Vega \& Javier Arroyo (2014) crearon un método llamado Smartick, el cual apoya de forma online a cada estudiante en aras de obtener avances satisfactorios en sus procesos matemáticos, adaptándose al estilo de aprendizaje de cada niño y ofreciendo un plan de estudio personalizado. La herramienta no realiza identificación de los estudiantes con dificultades de aprendizaje en la matemática, generando solo entrenamientos para mejorar la capacidad matemática.

- CogniFit (2015) creó una batería computarizada para la evaluación cognitiva de la discalculia o Cognitive Assessment Battery for Dyscalculia (CAB-DC). Dicha batería ayuda a detectar y analizar la presencia de algún síntoma, rasgos y dificultades en los procesos cognitivos relacionados con la discalculia. La persona realiza una serie de ejercicios y tareas validadas que se presentan en forma de sencillos juegos, donde se evalúan los dominios cognitivos (atención, memoria, coordinación, percepción, razonamiento y lenguaje), los resultados 
obtenidos deben ser revisados e interpretados por un profesional de la salud cualificado.

- Lancheros Cuesta, Carrillo Ramos, \& Pavlich Mariscal (2015) crean un módulo adaptativo, Kamachiy-Mayistru $(\mathrm{KM})$, para apoyar la enseñanza a personas con dificultades de aprendizaje, ayudando al docente en la preparación de un curso, teniendo en cuenta el perfil del alumno, el perfil de la dificultad de aprendizaje y las sugerencias de los modelos pedagógicos, con el fin de que el estudiante pueda utilizar la herramienta de forma que se ajuste a sus necesidades.

- Dinamo Números es un programa con el objetivo de dar apoyo a estudiantes en riesgo de desarrollar discalculia, ya que se obtienen resultados del nivel matemático de cada estudiante respecto a los otros estudiantes. La intervención es realizada solo para estudiantes que tienen retraso en el desarrollo matemático buscando evitar los efectos del desarrollo de la discalculia (DINAMO NÚMEROS, 2016).

- Velasquez Peñuela (2017) construyó una aplicación móvil llamada Discalapp, la cual permite mejorar el aprendizaje de los niños diagnosticados con dificultades específicas de aprendizaje, por medio de terapia del juego y actividades lúdicas. La herramienta está dividida en 4 módulos que le ayudan a adaptarse de acuerdo a la necesidad del niño.

Para el análisis de las herramientas tecnológicas revisadas se definieron cuatro componentes orientados a desarrollar apoyo adecuado a los estudiantes con DAM en el aula de clase, de acuerdo a lo revisado en el estado del arte. En la Tabla 1 se presenta la lista de herramientas tecnológicas y los componentes asociados. 
Tabla 1 - Herramienta tecnológicas evaluadas.

\begin{tabular}{|c|c|c|c|c|}
\hline $\begin{array}{l}\text { Herramientas } \\
\text { Tecnológicas }\end{array}$ & $\begin{array}{c}\text { Identificación } \\
\text { DAM }\end{array}$ & $\begin{array}{c}\text { Inter- } \\
\text { vención } \\
\text { DAM }\end{array}$ & $\begin{array}{c}\text { Evalu- } \\
\text { ación } \\
\text { procesos } \\
\text { cognitivos }\end{array}$ & $\begin{array}{l}\text { Segui- } \\
\text { miento } \\
\text { por el } \\
\text { docente }\end{array}$ \\
\hline $\begin{array}{c}\text { MULTIPLATA- } \\
\text { FORMA DE RE- } \\
\text { CURSOS EDUCA- } \\
\text { TIVOS } \\
\end{array}$ & & $\mathrm{X}$ & & \\
\hline SMARTICK & & $\mathrm{X}$ & $\mathrm{X}$ & $\mathrm{X}$ \\
\hline CAB-DC & $\mathrm{X}$ & $\mathrm{X}$ & & $\mathrm{X}$ \\
\hline $\begin{array}{l}\text { KAMACHIY } \\
\text {-MAYISTRU }\end{array}$ & $\mathrm{X}$ & & $\mathrm{X}$ & \\
\hline $\begin{array}{c}\text { DINAMO NÚME- } \\
\text { ROS } \\
\end{array}$ & $\mathrm{X}$ & $\mathrm{X}$ & & $\mathrm{X}$ \\
\hline DISCALAPP & $\mathrm{X}$ & $\mathrm{X}$ & & $\mathrm{X}$ \\
\hline
\end{tabular}

Fuente: Elaboración propia.

Estas revisiones de los componentes incluidos sirven de base para los requerimientos a una herramienta tecnológica orientada al apoyo real en el aula de clase tanto al estudiante como al docente.

Los componentes revisados son: La identificación DAM e intervención DAM se centran en cada procedimiento que realizan las pruebas: una identificación de las dificultades específicas de aprendizaje de la matemática que permita la detección del estudiante que requiere refuerzo en el proceso de aprendizaje en la matemática, y una la intervención adecuada para los niños identificados con dificultades específicas de aprendizaje de la matemática, utilizando aspectos relevantes para llegar a un avance satisfactorio en este ámbito. Evaluación de procesos cognitivos a la estimación de los 
procedimientos que lleva a cabo el estudiante para el desarrollo de las actividades propuestas: procesos como la atención, la memoria, el lenguaje, etc. Por último, el seguimiento asociado con el proceso de apoyo a estos estudiantes con DAM a causa de la intervención del docente.

Como se observa en la Tabla 1, es común en las herramientas la opción de identificar las DAM y ofrecer actividades para su intervención, pero menos en la evaluación concretar de los procesos cognitivos asociados ni en las herramientas de seguimiento posterior a la intervención. Estos aspectos son recogidos en el modelo y plataforma tecnología propuesta.

\section{Modelo para atención a niños con DAM}

\section{a. Modelo Conceptual}

En los procesos de enseñanza y aprendizaje de las matemáticas los estudiantes van avanzando a medida que realizan diversas actividades académicas y lúdicas (PÉREZ JIMENO, 2002). A partir de esta afirmación y teniendo en cuenta los componentes reconocidos en la revisión de trabajos relacionados, se propone un modelo centrado en actividades de aprendizaje, orientado a la identificación de las DAM de forma temprana en el aula de clase y que permita realizar un posterior apoyo a los estudiantes y docentes.

La Figura 1 expone gráficamente los diferentes componentes del modelo.

El modelo se compone de la prueba $\mathbf{D A M}$ que luego del análisis automático de los resultados permite la identificación $\boldsymbol{D A} \boldsymbol{M}$ determinado las habilidades y niveles en que el estudiante tiene debilidades y fortalezas. Como consecuencia, se propone un plan de trabajo orientado a mejorar las competencias en las áreas con mayor debilidad y que sirve de apoyo al docente para el acompañamiento necesario a este tipo de estudiantes. Con el fin de continuar con el proceso de evaluación de las condiciones específicas buscando conocer la evolución del estudiante a partir 
Figura 1 - Modelo Propuesto.

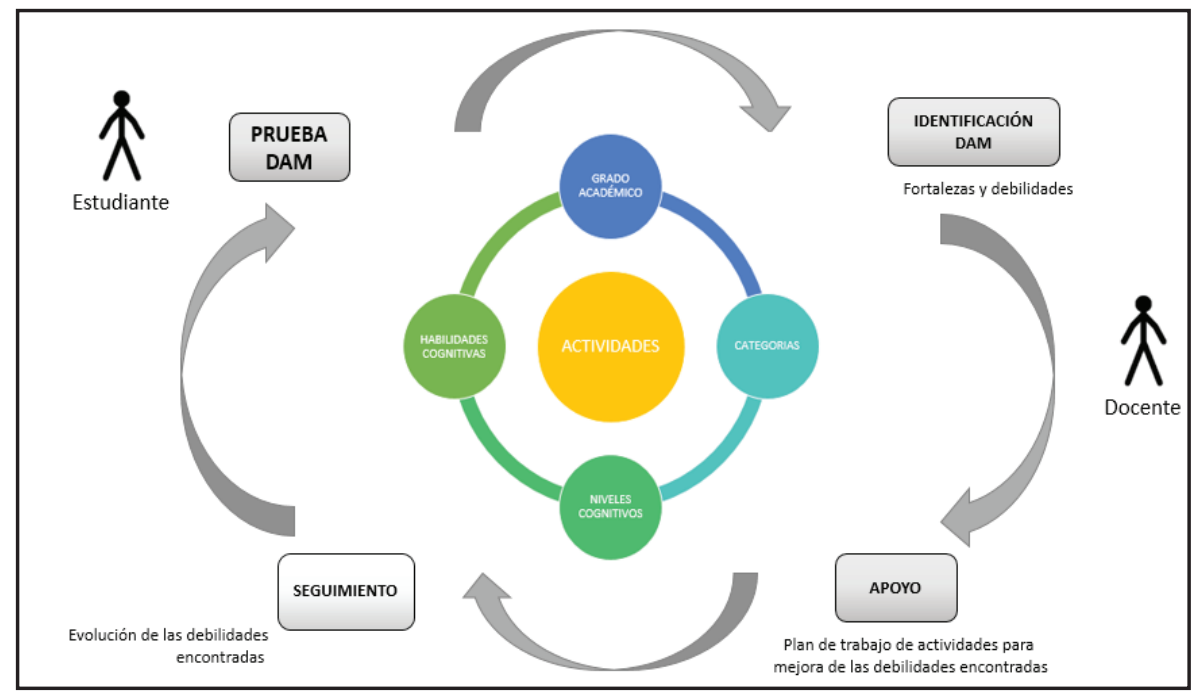

Fuente: Elaboración propia.

de las actividades propuestas, se tiene un componente de seguimiento a los estudiantes identificados.

Un factor importante en el modelo es poder definir o asociar las actividades de aprendizaje con diferentes aspectos tales como los niveles cognitivos, habilidades cognitivas y grado académico.

El grado académico está determinado por entidad que regula o determina los logros esperados en cada grado, generalmente los ministerios o secretarias de educación. Los niveles cognitivos definidos, según la revisión de la literatura, son: espacial, temporal, simbólico y cognitivo, que reflejan la manera de realizar un razonamiento lógico matemático y que permiten obtener esta información en el desarrollo de cada actividad. Las habilidades cognitivas definen el conocimiento requerido para la solución de cada actividad y para este modelo están asociadas al razonamiento numérico, razonamiento lógico, razonamiento espacial, razonamiento temporal, razonamiento visual, atención y funciones ejecutivas (memoria de trabajo, memoria de trabajo verbal, memoria de trabajo viso-espacial, inhibición, flexibilidad, planificación) (ACOSTA \& MIRANDA, 2012; ARRIETA, 2003; RIVIERE, 1990; MIGUEL POZA, 2016). 
Cuando el estudiante realiza la prueba, el diseño y definición de las actividades, debe permitir la identificación temprana de las DAM, lo que ayuda al docente a determinar las condiciones concretas que tienen sus estudiantes y como efecto el sistema debe definir el plan de actividades sugerido, que será la guía para el apoyo en la atención a las debilidades en los niveles cognitivos y en las habilidades matemáticas. El seguimiento permanente a los estudiantes con DAM se basa en observar los resultados y determinar si existe algún cambio de dichas debilidades. Es necesario que el estudiante realice nuevamente, en un tiempo prudente, la prueba para la identificación de las dificultades específicas de aprendizaje.

La versatilidad del modelo permite adicionar elementos de importancia a cada uno de los componentes para mejorar los mecanismos para la identificación temprana, el apoyo al docente, el seguimiento y sin duda la evaluación DAM.

Las Tablas 2 a la 4 muestran las relaciones entre estos componentes.

Tabla 2 - Actividades para los diferentes niveles según el grado académico.

\begin{tabular}{|c|c|c|c|c|}
\hline Grado & Nivel Espacial & Nivel Temporal & $\begin{array}{c}\text { Nivel } \\
\text { Simbólico }\end{array}$ & $\begin{array}{c}\text { Nivel } \\
\text { Cognitivo }\end{array}$ \\
\hline $\begin{array}{l}\text { Grado } \\
\text { Primero }\end{array}$ & $\begin{array}{l}\text { *Dictado de } \\
\text { números } \\
\text { *Figuras } \\
\text { geométricas } \\
\text { *Ubicación } \\
\text { izquierda/derecha } \\
\text { *Situación en el } \\
\text { espacio } \\
\text { *Seriación y } \\
\text { clasificación } \\
\text { *Conjuntos } \\
\text { *Ubicación en el } \\
\text { plano } \\
\text { Números espejo }\end{array}$ & $\begin{array}{l}\text { *Secuencias } \\
\text { temporales } \\
\text { *Secuencias } \\
\text { Rutinas diarias } \\
\text { *Días de la } \\
\text { semana } \\
\text { *Número anterior } \\
\text { y posterior } \\
\text { *Memorización }\end{array}$ & $\begin{array}{l}\text { *Símbolos } \\
\text { matemáti- } \\
\text { cos }\end{array}$ & $\begin{array}{l}\text { *Objetos } \\
\text { tachados } \\
\text { *Operaciones } \\
\text { matemáticas } \\
\text { *Problemas } \\
\text { matemáticos } \\
\text { *Escribir en } \\
\text { letra el número } \\
\text { *Escribir en } \\
\text { cifras el número } \\
\text { *Selecciona el } \\
\text { billete } \\
\text { *Número mayor } \\
\text { y menor }\end{array}$ \\
\hline
\end{tabular}


continuação Tabla 2 - Actividades para los diferentes niveles según el grado académico.

\begin{tabular}{|c|c|c|c|c|}
\hline Grado & Nivel Espacial & Nivel Temporal & $\begin{array}{c}\text { Nivel } \\
\text { Simbólico }\end{array}$ & $\begin{array}{c}\text { Nivel } \\
\text { Cognitivo }\end{array}$ \\
\hline $\begin{array}{l}\text { Grado } \\
\text { Segundo }\end{array}$ & $\begin{array}{l}\text { *Dictado de } \\
\text { números } \\
\text { *Figuras } \\
\text { geométricas } \\
\text { *Ubicación } \\
\text { izquierda/derecha } \\
\text { *Situación en el } \\
\text { espacio } \\
\text { *Seriación y } \\
\text { clasificación } \\
\text { *Conjuntos } \\
\text { *Ubicación } \\
\text { en el plan }\end{array}$ & $\begin{array}{l}\text { *Secuencias } \\
\text { temporales } \\
\text { *Secuencias } \\
\text { Rutinas diarias } \\
\text { *Días de la } \\
\text { semana y meses } \\
\text { del año } \\
\text { *Número } \\
\text { anterior y } \\
\text { posterior } \\
\text { *Memorización } \\
\text { El reloj }\end{array}$ & $\begin{array}{l}\text { *Símbolos } \\
\text { matemáti- } \\
\text { cos }\end{array}$ & $\begin{array}{l}\text { *Operaciones } \\
\text { matemáticas } \\
\text { *Problemas } \\
\text { matemáticos } \\
\text { *Escribir en } \\
\text { letra el número } \\
\text { *Escribir en } \\
\text { cifras el número } \\
\text { *Valor } \\
\text { posicional } \\
\text { Ordenar objetos }\end{array}$ \\
\hline
\end{tabular}

Tabla 3 - Asociación categorías de las actividades con los niveles cognitivos y habilidades cognitivas.

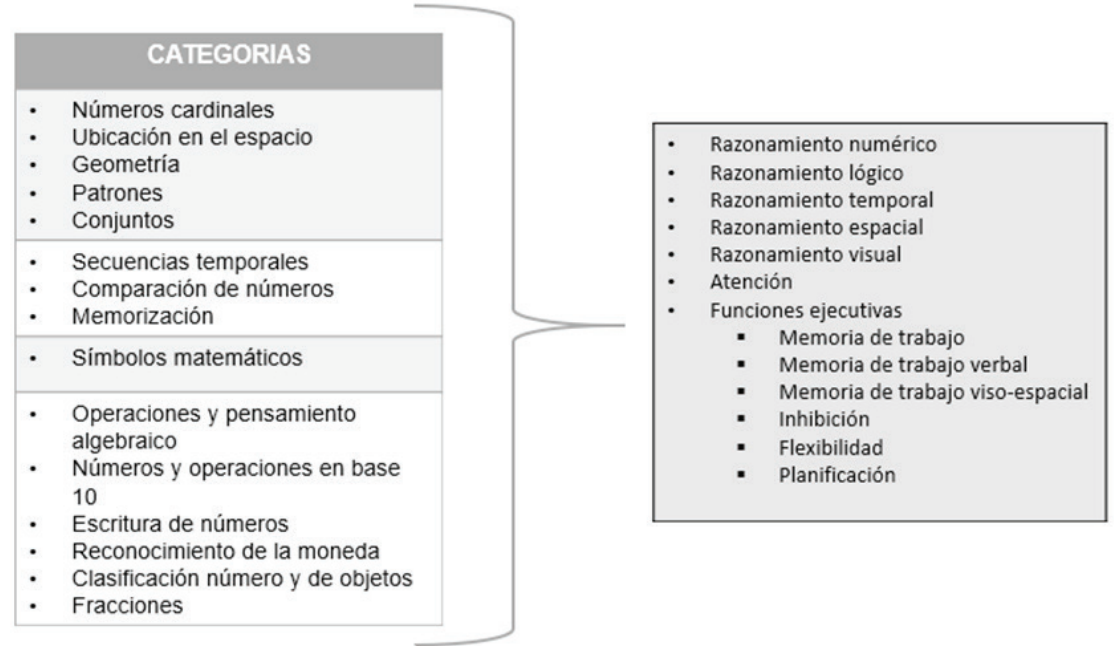


Tabla 4 - Ejemplo de actividades relacionadas con categorías y habilidades cognitivas para el nivel espacial.

\begin{tabular}{|c|c|c|c|}
\hline \multicolumn{4}{|c|}{ GRADO PRIMERO } \\
\hline \multirow{5}{*}{$\begin{array}{c}\text { NIVEL } \\
\text { ESPACIAL }\end{array}$} & ACTIVIDAD & CATEGORÍAS & $\begin{array}{c}\text { HABILIDADES } \\
\text { COGNITIVAS }\end{array}$ \\
\hline & $\begin{array}{l}\text { Elegir la forma } \\
\text { correcta }\end{array}$ & $\begin{array}{l}\text { Ubicación en } \\
\text { el espacio - } \\
\text { Geometría }\end{array}$ & $\begin{array}{l}\text { Razonamiento lógico - } \\
\text { Razonamiento espacial } \\
\text { - atención- memoria de } \\
\text { trabajo viso-espacial }\end{array}$ \\
\hline & $\begin{array}{l}\text { Serie simple } \\
\text { con uso de fig- } \\
\text { uras geométri- } \\
\text { cas }\end{array}$ & $\begin{array}{l}\text { Patrones - } \\
\text { Geometría - seria- } \\
\text { ción y clasificación }\end{array}$ & $\begin{array}{l}\text { razonamiento lógico - } \\
\text { razonamiento visual, } \\
\text { memoria de trabajo } \\
\text { viso-espacial }\end{array}$ \\
\hline & $\begin{array}{l}\text { ¿Cuántos lados } \\
\text { tiene la figura? }\end{array}$ & $\begin{array}{l}\text { Ubicación en } \\
\text { el espacio - } \\
\text { Geometría }\end{array}$ & $\begin{array}{l}\text { Razonamiento lógico - } \\
\text { Razonamiento espacial } \\
\text { - atención- memoria de } \\
\text { trabajo viso-espacial }\end{array}$ \\
\hline & $\begin{array}{l}\text { Elegir el objeto } \\
\text { que está lejos, } \\
\text { encima y detrás } \\
\text { del objetivo }\end{array}$ & $\begin{array}{l}\text { Ubicación en el } \\
\text { espacio }\end{array}$ & $\begin{array}{l}\text { Razonamiento espacial- } \\
\text { razonamiento lógico } \\
\text { - memoria de trabaja } \\
\text { viso-espacial }\end{array}$ \\
\hline
\end{tabular}

\section{b. Modelo tecnológico}

El entorno informático para la identificación y apoyo a niños con dificultades de aprendizaje de la matemática es denominado Diamante, a partir de la unión de palabras de importancia como diagnóstico, identificación, aptitud, matemática, estudiante. Dicho entorno fue desarrollado tomando en cuenta el modelo conceptual. Este entorno está orientado a ser una herramienta de apoyo a los docentes en el aula de clase para realizar una identificación de posibles DAM de sus estudiantes y así mismo obtener resultados que ayuden a realizar un plan de trabajo buscando mejorar el proceso de enseñanza y aprendizaje. 
Diamante es un entorno informático desarrollado con herramientas libres enfocado a la Web, con la ventaja de poder ser instalado en diversos ambientes y estar disponible en la red y con el requerimiento básico a profesores y estudiantes de un navegador web. Esta herramienta puede llegar a convertirse en un gran aliado para las instituciones educativas y también para los docentes para realizar una identificación de DAM de una forma sencilla y permitiendo hacer el seguimiento tanto individual como grupal a sus estudiantes. El modelo está orientado a actividades y apoya en la identificación temprana de las DAM, y a la vez ofrece actividades de aprendizaje en línea, orientadas a enfrentar las falencias de los estudiantes que tienen dificultades.

La Figura 2 muestra la página principal del entorno informático mediante la cual se accede a los diferentes módulos, dependiendo del tipo de usuario.

Figura 2 - Página inicial Diamante.

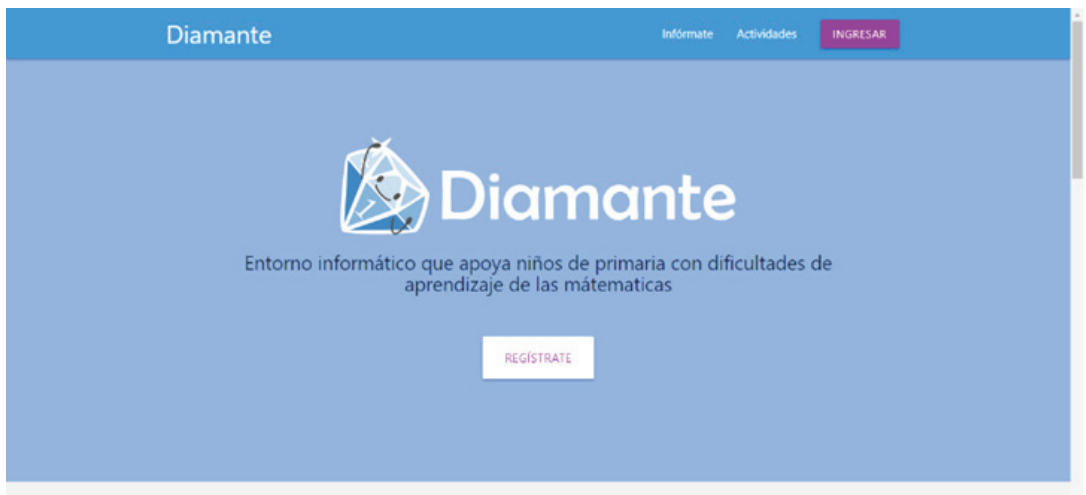

Las Figuras 3, 4 y 5 presentan diferentes actividades para los grados 1,2 y 3 . 
Figura 3 - Actividades para los diferentes niveles cognitivos para el Grado primero.

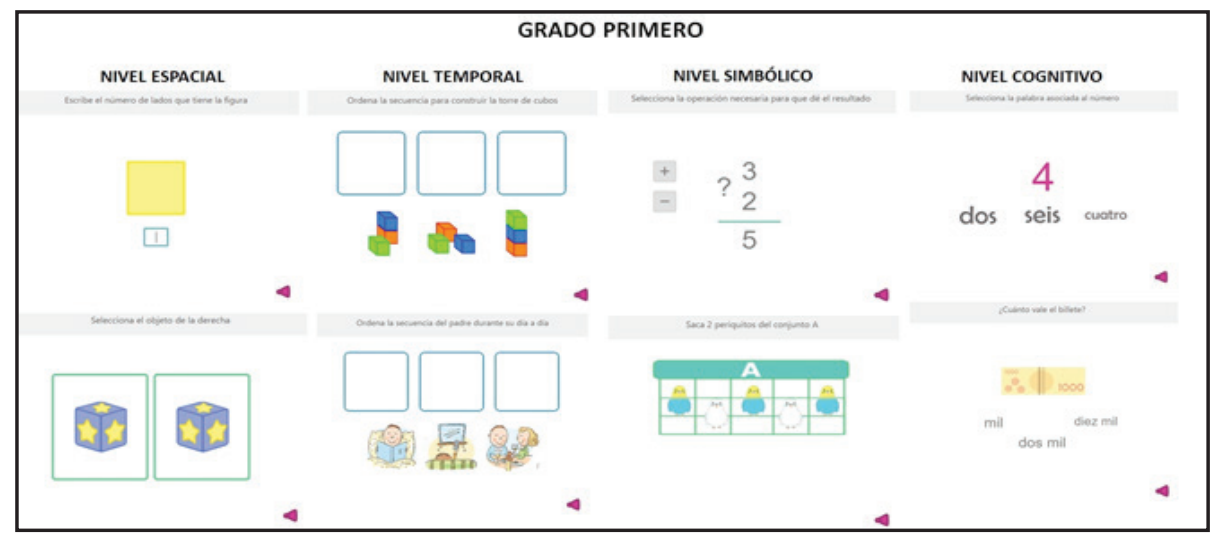

Figura 4 - Actividades para los diferentes niveles cognitivos para el Grado segundo.

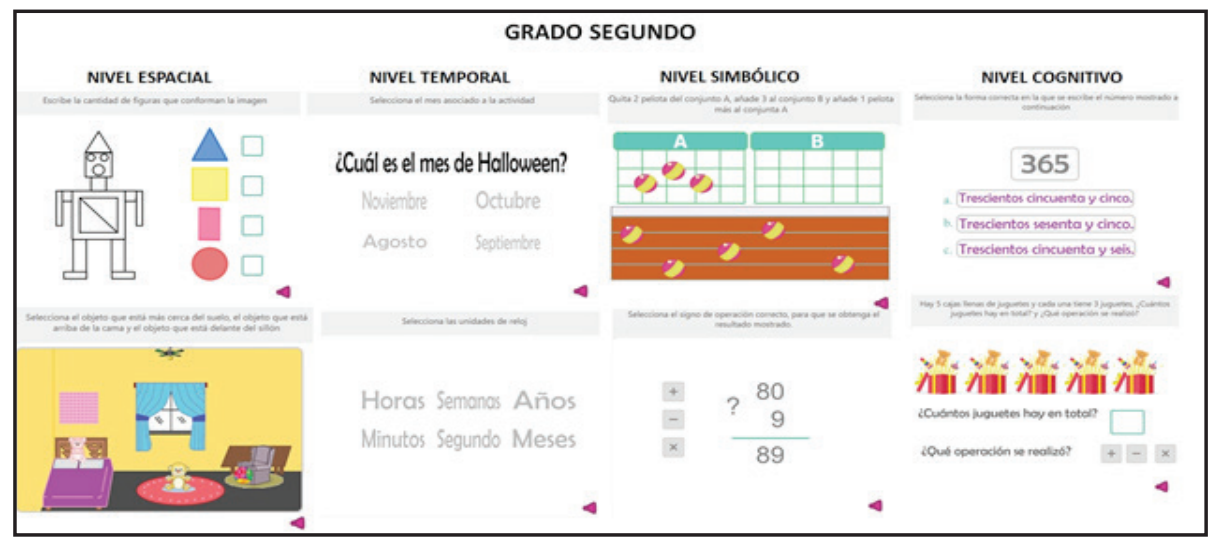

\section{c. Validación del entorno "Diamante"}

Las actividades a desarrollar se definieron de acuerdo con los derechos básicos de aprendizaje de la matemática del Ministerio de Educación Nacional de Colombia, 2016 retomando las temáticas y/o las habilidades que debe adquirir un estudiante de grado primero, segundo y tercero de primaria durante su proceso de aprendizaje.

Se realizó un proceso de validación del entorno informático educativo por medio de 9 estudiantes del colegio Aspaen Los Cerezos de la ciudad de Manizales- 
Colombia del grado primero de primaria y 5 estudiantes como casos individuales de otros colegios. Se obtuvo las respuestas de cada uno de acuerdo a cada nivel. Para este caso, cada nivel tenía 5 actividades, en total fueron 20 actividades por estudiante. Estas son seleccionadas por el entorno de forma aleatoria desde la base de datos del repositorio completo con actividades de todas las temáticas.

Figura 5 - Actividades para los diferentes niveles cognitivos para el Grado tercero.

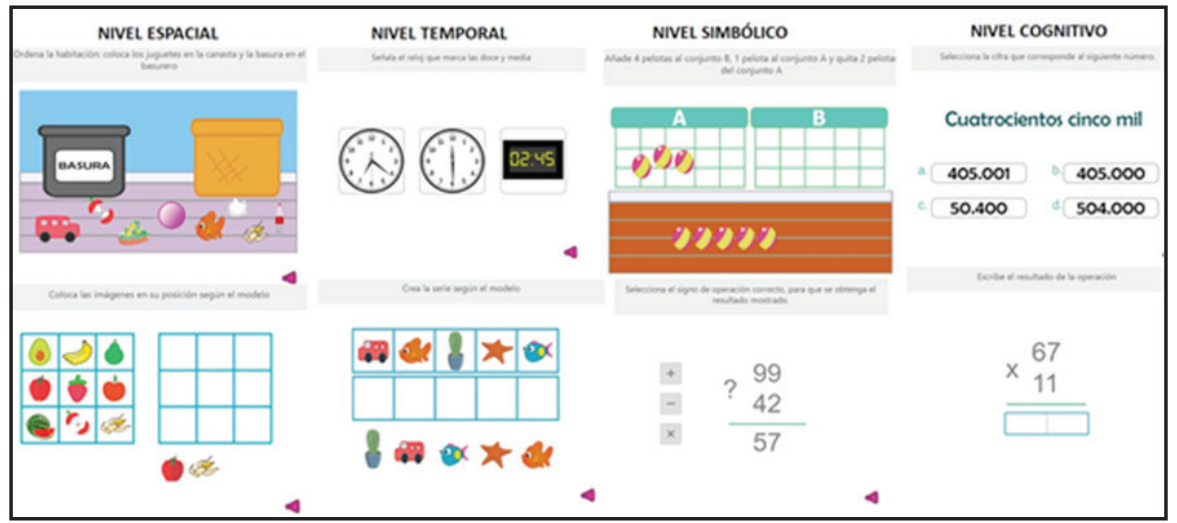

Las Tabla 5 y 6 muestran las respuestas de cada uno de los estudiantes, para observar de forma individual su proceso en la prueba DAM y permitir de forma más sencilla la identificación de las debilidades y fortalezas de cada uno.

Tabla 5 - Resultados estudiantes colegio Aspaen los cerezos.

\begin{tabular}{c|c|c|c|c|c|c}
\hline Estudiante & GRUPO & NE $\%$ & NT $\%$ & NS $\%$ & NC $\%$ & Puntaje Total \% \\
\hline $\mathbf{1}$ & 1 & 0,7 & 0,2 & 0,6 & 1,0 & 0,6 \\
\hline $\mathbf{2}$ & 1 & 0,9 & 0,2 & 0,6 & 1,0 & 0,7 \\
\hline $\mathbf{3}$ & 1 & 1,0 & 0,4 & 0,8 & 1,0 & 0,8 \\
\hline $\mathbf{4}$ & 1 & 1,0 & 0,7 & 0,8 & 1,0 & 0,9 \\
\hline $\mathbf{5}$ & 1 & 1,0 & 0,7 & 0,4 & 0,4 & 0,6 \\
\hline $\mathbf{6}$ & 1 & 1,0 & 0,8 & 0,6 & 1,0 & 0,9 \\
\hline $\mathbf{7}$ & 1 & 1,0 & 0,9 & 0,8 & 1,0 & 0,9 \\
\hline $\mathbf{8}$ & 1 & 0,7 & 0,9 & 1,0 & 0,6 & 0,8 \\
\hline $\mathbf{9}$ & 1 & 0,5 & 1,0 & 0,4 & 0,6 & 0,6 \\
\hline
\end{tabular}


Tabla 6 - Resultados casos individuales.

\begin{tabular}{c|c|c|c|c|c}
\hline Estudiante & NE $\%$ & NT \% & NS \% & NC \% & Puntaje Total \% \\
\hline $\mathbf{1}$ & 1,0 & 0,8 & 1,0 & 1,0 & 0.95 \\
$\mathbf{2}$ & 1,0 & 0,73 & 0,8 & 0,8 & 0,83 \\
\hline $\mathbf{3}$ & 1,0 & 0,87 & 0,8 & 1,0 & 0,92 \\
\hline $\mathbf{4}$ & 1,0 & 0,87 & 1,0 & 1,0 & 0,97 \\
\hline $\mathbf{5}$ & 1,0 & 1,0 & 1,0 & 1,0 & 1,0 \\
\hline
\end{tabular}

Como se puede apreciar, para el caso bajo análisis, la prueba no identifica ningún estudiante con dificultades de aprendizaje, pero sí estudiantes que tienen alguna debilidad en un nivel específico, lo que requiere realizar un plan de trabajo de acuerdo a la falencia encontrada.

Al conocer el rendimiento de los alumnos y también de los grupos, se puede profundizar y reflexionar sobre aspectos que se encuentran relacionados con las características concretas de sus dificultades, esto ha de verse reflejado de forma favorable en una atención pedagógica que el profesor puede ofrecerles a sus alumnos, considerándolo tanto individual como grupal, promoviendo de forma continua la búsqueda de estrategias para mejorar la practica como docente (ETCHEPAREBORDA \& ABADMAS, 2005)mediante procesos neurobiológicos de almacenamiento y de recuperación de la información, básica en el aprendizaje y en el pensamiento. En los primeros años de la vida, la memoria es de carácter sensitivo, guarda sensaciones o emociones. Más tarde aparece la memoria de las conductas: se ensayan movimientos, se repi-ten y, poco a poco, se van grabando. De esa forma, los niños van reteniendo y aprendiendo experiencias que permiten que pro-grese y se adapte al entorno. Finalmente, se desarrolla la memo-ria del conocimiento, o capacidad de introducir datos, almace-narlos correctamente y evocarlos cuando sea oportuno. El sistema de la memoria está integrado por tres procesos básicos: - Codificación de la información. La codificación o adquisi-ción es el proceso en donde se prepara la información para que se pueda guardar. La información puede codificarse como una imagen, sonidos, experiencias, acontecimientos o ideas significativas. Las circunstancias que rodean este mo-mento resultan fundamentales para el éxito o fracaso 
de la memoria. Es importante en este procesos inicial, la aten-ción, la concentración y el estado emocional del sujeto. - Almacenamiento de la información. Esta etapa se caracteri-za por el ordenamiento, categorización o simple titulación de la información mientras se desarrolla el proceso en curso (proceso perfuncional(MCGUIRE, KINZIE, THUNDER, \& BERRY, 2014).

\section{Conclusiones y trabajo futuro}

De la revisión del estado del arte se puede afirmar que en el ambiente escolar existen estudiantes con diferentes dificultades específicas de aprendizaje, en particular en las matemáticas, y el docente debe enfrentar esta situación sin contar con los conocimientos suficientes ni con herramientas que lo apoyen en la detección y posterior apoyo a estos niños.

Los resultados obtenidos en las pruebas permiten concluir que los estudiantes tienen dificultades, casi siempre, en algunos de los niveles cognitivos, mientras que en otros no se presente esta situación, lo que exige que las estrategias estén orientadas en forma personalizada a enfrentar este hecho.

La implementación de la prueba ha permitido identificar las fortalezas y las debilidades de acuerdo a los elementos evaluados, teniendo como gran protagonista los 4 niveles cognitivos definidos a las cuales se sujetan las actividades: Nivel Espacial, Nivel Temporal, Nivel Simbólico y Nivel Cognitivo.

Las funcionalidades de Diamante y los reportes que ofrece al docente convierten esta plataforma en un aliado disponible para los profesores en los procesos para la detección, evaluación y seguimiento a los estudiantes con dificultades de aprendizaje de las matemáticas, mediante actividades de aprendizaje.

Como trabajo futuro, se traza el crecimiento del modelo realizando una modificación en el elemento grado académico, para así poder evaluar estudiantes que se encuentren en grados superiores a los evaluados con la herramienta. También se espera poder realizar la adición de habilidades cognitivas para realizar una identificación mucho más detallada. 
Se espera definir los mecanismos para que Diamante pueda ser aprovechado por diferentes instituciones educativas en forma libre y así ayudar a los docentes en la identificación de las DAM y aprovechar al máximo esta herramienta para apoyo en estrategias educativas.

\section{Bibliografía}

ACOSTA, G., \& MIRANDA, A. (2012). Evolución del funcionamiento ejecutivo en alumnos con y sin dificultades de aprendizaje en la resolución de problemas matemáticos. Un estudio longitudinal.

ANDALUCÍA, F. de E. de C. O. de. (2012). Dificultades de aprendizaje de la matemáticas. Revista Digital Para Profesionales de La Enseñanza, 20 (1990).

ARRIETA, M. (2003). Capacidad espacial y educación matemática: tres problemas para el futuro de la investigación. Educación Matemática, 15 (3), 57-76.

BLANCO PÉREZ, M. (2009). Dificultades específicas del aprendizaje de las matemáticas en los primeros años de la escolaridad. detección precoz y características evolutivas. Ministerio de Educacion - España.

BUTTERWORTH, B. (2011). Foundational Numerical Capacities and the Origins of Dyscalculia. Space, Time and Number in the Brain, 14 (12), 249-265. https://doi.org/10.1016/B978-0-12-385948-8.00016-5

COGNIFIT (2015). Batería de Evaluación Neuropsicológica Online. Instrumentos de ayuda al diagnóstico en Discalculia (CAB-DC).

DÍAZ, C. (2014). Dificultades de aprendizaje en las matemáticas, prevención y actuación (January).

DINAMO NÚMEROS (2017). Dyscalculia Profiler for Low Math Achievers. Retrieved from www.dynamoprofiler.com/

ETCHEPAREBORDA, M. C., \& ABAD-MAS, L. (2005). Memoria de trabajo en los procesos básicos del aprendizaje. Revista Neurologia, 40 (Supl 1), 79-83.

GARCÍA MORENO, Juan (2010). Así calculamos en mi cole.

GONZÁLEZ DE VEGA, Daniel, \& JAVIER ARROYO (2014). SmartClick.

IGLESIAS SARMIENTO, V. (2008). Dificultades de aprendizaje en el dominio aritmético y en el procesamiento cognitivo subyacente, Ph.D. (July 2008), 440.

LANCHEROS CUESTA, D., CARRILLO RAMOS, A., \& PAVLICH MARISCAL, J. A. (2015). Kamachiy- Mayistru: adpative module to support teaching to people 
with learning difficulties. Internacional Journal of Web Information Systems, 11, 510-526.

MCGUIRE, P. R., KINZIE, M., THUNDER, K., \& BERRY, R. (2014). Methods of Analysis and Overall Mathematics Teaching Quality in At-Risk Prekindergarten Classrooms. Early Education and Development, 9289 (March). https://doi.or $\mathrm{g} / 10.1080 / 10409289.2015 .968241$

MIGUEL POZA, P. (2016). Propuesta de intervención en el aula para desarrollar las capacidades cognitivas básicas a través de las matemáticas.

MINISTERIO DE EDUCACIÓN NACIONAL DE COLOMBIA (2016). Derechos básicos de Aprendizaje.

PÉREZ JIMENO, M. (2002). Problemas y dificultades en el aprendizaje matemático de los niños y niñas de tercer ciclo de primaria. Al Otro Lado de Las Fronteras de Las Matemáticas Escolares.

RAPIN, I. (2016). Dyscalculia and the Calculating Brain. Pediatric Neurology, 61, 11-20. https://doi.org/10.1016/j.pediatrneurol.2016.02.007

RIVIERE, Ä. (1990). Problemas y dificultades en el aprendizaje de las matemáticas: una perspectiva cognitiva. In: Desarrollo psicológico y educación, III. Necesidades educativas especiales y aprendizaje escolar (p. 155-182). Madrid (España): Alianza.

ROMERO PÉREZ, J. F., \& LAVIGNE CERVÁN, R. (2004). Dificultades en el Aprendizaje II: Unificación de Criterios Diagnósticos.

RUBO, A. M. P. (2007). Los procesos de exclusión en el ámbito escolar: el fracaso escolar y sus actores. Universidad Nacional Del Nordeste.

SEVILLANO, P., \& ANGELES, M. (2000). Tecnologías de apoyo a la discapacidad y dificultades de aprendizaje. Comunicar (15), 159.

VELASQUEZ PEÑUELA, N. (2017). DISCALAPP, Herramientas de apoyo para tratar dislexia y discalculia en niños de 5 a 7 años.

Submetido em: 9-10-2018

Aceito em: 23-11-2018

EDUCAÇÃO \& LINGUAGEM • V. $21 \bullet$ N. 2 • 141-161 • JUL.-DEZ. 2018 